\title{
Retrospective analysis of genetic abnormalities and survival in 131 patients with multiple myeloma
}

\author{
NIAN LIU, HEBING ZHOU, GUANGZHONG YANG, CHUANYING GENG, \\ YUAN JIAN, HUAN GUO and WENMING CHEN \\ Department of Hematology, Beijing Chaoyang Hospital, Capital Medical University, Beijing 100020, P.R. China
}

Received January 14, 2014; Accepted September 18, 2014

DOI: $10.3892 / \mathrm{ol} .2014 .2750$

\begin{abstract}
Genetic abnormalities in patients with multiple myeloma (MM) are important risk factors in terms of prognosis. In the present study, the prognostic value of several common MM genetic abnormalities was investigated. Interphase fluorescence in situ hybridization (iFISH) was used to detect genetic abnormalities, including 1q21 gain, $\mathrm{t}(4 ; 14)$, $\mathrm{t}(11 ; 14), \mathrm{t}(14 ; 16)$ and $17 \mathrm{p} 13$ deletion in 131 patients. A total of $46.6 \%$ patients were detected with one or more abnormalities using iFISH analysis. The 1q21 gain, $\mathrm{t}(4 ; 14), \mathrm{t}(11 ; 14), \mathrm{t}(14 ; 16)$ and $17 \mathrm{p} 13$ deletion abnormalities were detected in $42.5,6.9$, $17.5,0.8$ and $10.7 \%$ of patients, respectively. Patients with $\mathrm{t}(4 ; 14)$ commonly exhibited lower levels of albumin and hemoglobin. The progression-free survival (PFS) and overall survival times of iFISH-positive patients (particularly patients with two or more iFISH abnormalities) were significantly shorter than those of the patients without detectable abnormalities. The 1q21 gain and 17p13 deletion were also adverse prognostic factors for MM. Bortezomib-based therapies improved the PFS times in the patients with unfavorable iFISH abnormalities. These findings demonstrate that patients with two or more iFISH abnormalities, a gain of the 1q21 region or a $17 \mathrm{p} 13$ deletion were more likely to have a poor prognosis; however, bortezomib treatment improved the outcome for MM patients with unfavorable iFISH abnormalities.
\end{abstract}

\section{Introduction}

Multiple myeloma (MM) is characterized by the clonal proliferation of plasma cells and the subsequent accumulation of these cells within the bone marrow. The prognosis of patients with MM is variable, with survival times ranging between a few months and $>10$ years (1). Despite the recent

Correspondence to: Professor Wenming Chen, Department of Hematology, Beijing Chaoyang Hospital, Capital Medical University, 8 Gongti South Road, Beijing 100020, P.R. China

E-mail: 13910107759@163.com

Key words: multiple myeloma, fluorescence in situ hybridization, genetic abnormalities, prognostic factors application of novel drugs in clinical practice, MM remains incurable. Studies with large samples have shown that molecular cytogenetic changes have an important role in the prognosis of MM $(2,3)$. MM patients with extra copies of 1q, del(13)(q14), t(4;14), t(14;16) or del(17)(p13) exhibit an unfavorable prognosis, while the $\mathrm{t}(11 ; 14)$ translocation is associated with a better outcome. The incidence rates of the aforementioned genetic abnormalities are as follows: 28.9$43.0 \%$ for 1q21 gain $(4,5) ; 48.0-49.6 \%$ for $\operatorname{del}(13)(q 14)(6,7)$; $11.0-17.0 \%$ for $\mathrm{t}(4 ; 14)(6,8-13) ; 3.0-4.6 \%$ for $\mathrm{t}(14 ; 16)(9,12,13)$; 9.5-33.8\% for $\operatorname{del}(17)(\mathrm{p} 13)(6,9,11,13-15)$; and $12.8-21.0 \%$ for $\mathrm{t}(11 ; 14)(6,9-12)$. Approximately a decade ago, combination chemotherapy regimens, such as melphalan-prednisone or vincristine-doxorubicin-dexamethasone combined with stem cell transplantation were the standard treatment modalities for MM. However, the introduction of newer therapies, including the proteasome inhibitor bortezomib and immunomodulatory drugs (thalidomide and lenalidomide) has significantly improved patient survival. Previous studies have also shown that bortezomib induction improves the outcome of newly diagnosed patients with $t(4 ; 14)(16-18)$. The main objective of the present study was to evaluate the frequency and prognostic impact of several common fluorescence in situ hybridization (iFISH) abnormalities in patients with MM.

\section{Patients and methods}

Patients. A total of 107 newly diagnosed patients and 24 relapsed MM patients from Beijing Chaoyang Hospital (Beijing, China) were included in the present study. The diagnostic criteria were primarily derived from those provided by the World Health Organization (19). The International Staging System (ISS) (20) and the Durie-Salmon (DS) staging (21) were employed to assess these patients. Among the 128 patients with available treatment information, 88 received bortezomib-based combination chemotherapy. This treatment was composed of bortezomib $\left(1.3 \mathrm{mg} / \mathrm{m}^{2}\right.$, days $1,4,8$ and 11) plus dexamethasone $(20 \mathrm{mg} /$ day, days $1,2,4,5,8,9,11$ and 12) or dexamethasone in combination with doxorubicin (9 mg/m², days 1-4), Cytoxan $\left(300 \mathrm{mg} / \mathrm{m}^{2}\right.$, days $1-4)$ or thalidomide (100 mg/day, days 1-21). The patients who received this regimen were administered a median of four treatment courses (1-7 three-week courses). The other 40 patients received non-bortezomib-based therapy, involving 
Table I. Characteristics of patients.

Characteristics

Median age, years (range)

Gender, n (\%)

Male

Female

Durie-Salmon stage, n (\%)

I

II

III

Durie-Salmon substage, n (\%)

$$
\text { A }
$$$$
\text { B }
$$

ISS stage, n (\%)

I

II

III

Ig isotype, $\mathrm{n}(\%)$

IgG

$\operatorname{IgA}$

$\operatorname{IgD}$

Light chain

No paraprotein

Median Hb level, g/dl (range)

Median calcium level, mmol/l (range)

Median CRP level, mg/l (range)

Median $\beta 2$-microgolbulin level, mg/l (range)

Median albumin level, g/l (range)

Treatment response, $\mathrm{n}(\%)$

CR

VGPR

PR

$<$ PR

Treatment, n (\%)

Bortezomib-based combination chemotherapy

Non-bortezomib-based therapy

ASCT, n (\%)

Yes

No
124

124

94

124

Value

$59(38-82)$

$74(56.5)$

57 (43.5)

114

$1(0.9)$

15 (13.1)

98 (86.0)

109

75 (68.8)

34 (31.2)

105

$11(10.5)$

39 (37.1)

55 (52.4)

129

124

122

59 (45.7)

33 (25.6)

$9(7.0)$

23 (17.8)

5 (3.9)

9.3 (4.4-16.6)

$2.18(1.25-3.83)$

0.4 (0.1-13.7)

$3.50(0.95-34.15)$

31.9 (15.2-46.5)

36 (29.5)

16 (13.1)

$51(41.8)$

19 (15.6)

128

88 (68.8)

40 (31.2)

131

108 (82.4)

ISS, International Staging System; Hb, hemoglobin; CRP, C-reactive protein; CR, complete response; VGPR, very good partial response; PR, partial response; ASCT, autologous stem cell transplantation.

either doxorubicin $\left(9 \mathrm{mg} / \mathrm{m}^{2}\right.$, days $\left.1-4\right)$, vincristine $(0.4 \mathrm{mg} /$ day, days 1-4) and dexamethasone (20 mg/day, days 1-4, 9-12 and 17-20), thalidomide (100 $\mathrm{mg} /$ day, day 1-28), doxorubicin $\left(9 \mathrm{mg} / \mathrm{m}^{2}\right.$, days 1-4) and dexamethasone $(20 \mathrm{mg} /$ day, days 1-4, 9-12 and 17-20), or melphalan (4 mg/m², days $1-7)$, prednisone $(60 \mathrm{mg} /$ day, days $1-7)$ and thalidomide (100 mg/day, days 1-28). The patients on this regime received a median of four treatment courses (1-8 four week courses). Following the induction of therapy, 23 patients received autologous stem cell transplantation and maintenance therapy, and the other 108 patients received only maintenance therapy. The efficacy assessment was conducted according to the International Myeloma Working Group criteria (22). The median follow-up time was 21.3 months (range, 0.2-109.3 months). The clinical and laboratory features of all 131 patients are summarized in Table I. This study was approved by the ethics committee of 
Table II. Response rate of multiple myeloma patients receiving bortezomib-based therapy or conventional therapy.

\begin{tabular}{|c|c|c|c|c|}
\hline & \multicolumn{2}{|c|}{ ORR, n (\%) } & \multicolumn{2}{|c|}{$\geq$ VGPR, n (\%) } \\
\hline & $\begin{array}{l}\text { Bortezomib-based } \\
\text { therapy }\end{array}$ & $\begin{array}{c}\text { Non-bortezomib-based } \\
\text { therapy }\end{array}$ & $\begin{array}{l}\text { Bortezomib-based } \\
\text { therapy }\end{array}$ & $\begin{array}{c}\text { Non-bortezomib-based } \\
\text { therapy }\end{array}$ \\
\hline Normal FISH & $41(95.3)$ & 15 (68.2) & $26(60.1)$ & $3(13.6)$ \\
\hline $\operatorname{del}(17)(\mathrm{p} 13)$ & $6(75.0)$ & $3(75.0)$ & $4(50.0)$ & $1(25.0)$ \\
\hline 1q21 gain & $20(87.0)$ & $8(72.7)$ & $11(47.8)$ & $2(18.2)$ \\
\hline $\mathrm{t}(4 ; 14)(\mathrm{p} 16 ; \mathrm{q} 32)$ & $4(100.0)$ & $3(75.0)$ & $4(100.0)$ & $0(0.0)$ \\
\hline $\mathrm{t}(11 ; 14)(\mathrm{q} 13 ; \mathrm{q} 32)$ & $7(70.0)$ & $2(66.7)$ & $1(10.0)$ & $1(33.3)$ \\
\hline
\end{tabular}

ORR, overall response rate; VGPR, very good partial response; FISH, fluorescence in situ hybridization.

Beijing Chaoyang Hospital and written informed consent was obtained from all patients.

Interphase FISH (iFISH) analysis. A 5-10-ml sample of bone marrow was obtained with informed consent from the patients with MM at the time of diagnosis or relapse, and was then mixed with heparin. Mononuclear cells were enriched by the Ficoll-gradient centrifugation method (Ficoll-Paque PLUS; GE Healthcare Bio-Sciences AB, Uppsala, Sweden), and then were assessed using commercially available probes for the regions containing 4p16 (FGFR3)/14q32 (IGH), 11q13 $(C C N D 1) / 14 \mathrm{q} 32(I G H), 14 \mathrm{q} 32(I G H) / 16 \mathrm{q} 32(M A F), 1 \mathrm{q} 21$ and 17p13.1 (TP53) using Vysis TP53/CEP 17 FISH, Vysis IGH/MAF DF FISH and Vysis IGH/CCND1 DF FISH Probe Kits, a Vysis LSI IGH/FGFR3 Dual Color, Dual Fusion Translocation Probe (Vysis/Abbott Molecular, Des Plaines, IL, USA) and a LSI 1q21 FISH Probe Kit (China Meditech, Beijing, China). Slides containing the cells were pretreated, denatured and hybridized using standard laboratory procedures following the manufacturer's instructions (Vysis/Abbott Molecular). A minimum of 500 mononuclear cells were scored for each iFISH signal to estimate the prevalence of each molecular cytogenetic abnormality. The positive cutoff level for each iFISH probe set was established as $>10 \%$.

Statistical analysis. Associations between genetic abnormalities and biological parameters were evaluated by independent samples t-test. Comparison of frequencies among groups was performed using the $\chi^{2}$ test. The overall survival (OS) and progression-free survival (PFS) time distributions were estimated using the Kaplan-Meier method, and differences between survival curves were analyzed using the log-rank test. PFS times were only evaluated in patients who achieved at least a partial response (PR). All statistical analyses were performed using SPSS version 18.0 (SPSS, Inc., Chicago, IL, USA). $\mathrm{P}<0.05$ was considered to indicate a statistically significant difference.

\section{Results}

Genetic abnormalities detected by iFISH. A total of 61 patients (46.6\%) exhibited abnormal iFISH results. Gain of 1q21 was the most common genetic abnormality, with an incidence of
$42.5 \%(34 / 80)$, whereas $t(14 ; 16)$ was the least common abnormality exhibited, with an incidence of $0.8 \%(1 / 131)$. The presence of the $17 \mathrm{p}$ deletion was detected in $10.7 \%(14 / 131)$ of cases. A total of $17.5 \%(14 / 80)$ of patients had $t(11 ; 14)$, whereas $6.9 \%(9 / 131)$ had $t(4 ; 14)$. Two cases $(2.5 \%)$ presented with three abnormalities, 18 cases $(22.5 \%)$ with two and 27 cases $(33.8 \%)$ with one. In total, of the 80 patients tested, 33 patients (41.3\%) did not exhibit any of the five abnormalities. Due to the small sample size, $t(14 ; 16)$ was not included in any further analysis.

Correlation between iFISH abnormalities and biological parameters. No significant differences in gender $(\mathrm{P}=0.648)$, age ( $\geq 65$ years vs. $<65$ years; $P=0.586$ ) and status of disease (newly diagnosed or relapsed; $\mathrm{P}=0.709$ ) were identified between patients with positive iFISH results and those without an abnormality. However, in comparison to patients with normal iFISH results, the patients with abnormal iFISH results exhibited significantly higher calcium levels (median, 2.26 vs. $2.14 \mathrm{mmol} / \mathrm{l} ; \mathrm{P}=0.002$ ), lower hemoglobin levels (median, 8.8 vs. $9.7 \mathrm{~g} / \mathrm{dl} ; \mathrm{P}=0.024$ ) and a higher DS stage $(\mathrm{P}=0.018)$. $\beta 2$-microglobulin and $\mathrm{C}$-reactive protein levels were also higher in the patients with abnormal iFISH results compared with the patients with normal iFISH results (median, 5.09 vs. $3.06 \mathrm{mg} / 1$ and 0.50 vs. $0.42 \mathrm{mg} / 1$, respectively), but these results were not statistically significant. In addition, in comparison to patients who lacked $t(4 ; 14)$, the patients with $\mathrm{t}(4 ; 14)$ had significantly lower albumin levels (median, 25.0 vs. $32.6 \mathrm{~g} / 1 ; \mathrm{P}=0.008$ ) and lower hemoglobin levels (median 7.6 vs. $9.5 \mathrm{~g} / \mathrm{dl} ; \mathrm{P}<0.001$ ).

Correlation between iFISH abnormalities and response rates. A total of 122 patients had best treatment response assessment results available. The overall response rate (ORR; PR or better) of treatment was $84.4 \%$ (103/122) and a very good partial response (VGPR) or better was achieved in $42.6 \%(52 / 122)$ patients. The 65 patients who had no detectable abnormalities exhibited an ORR of $86.2 \%(56 / 65)$, a rate comparable with that of patients with iFISH abnormalities $(82.5 \%$; 47/57). Similarly, no significant differences in ORR were observed between the patients with versus those without del(17p) (75.0 vs. 85.5\%; $\mathrm{P}=0.597), \mathrm{t}(11 ; 14)(69.2$ vs. $82.0 \% ; \mathrm{P}=0.511), \mathrm{t}(4 ; 14)(87.5$ vs. $84.2 \% ; \mathrm{P}=1.000)$ and $1 \mathrm{q} 21$ 

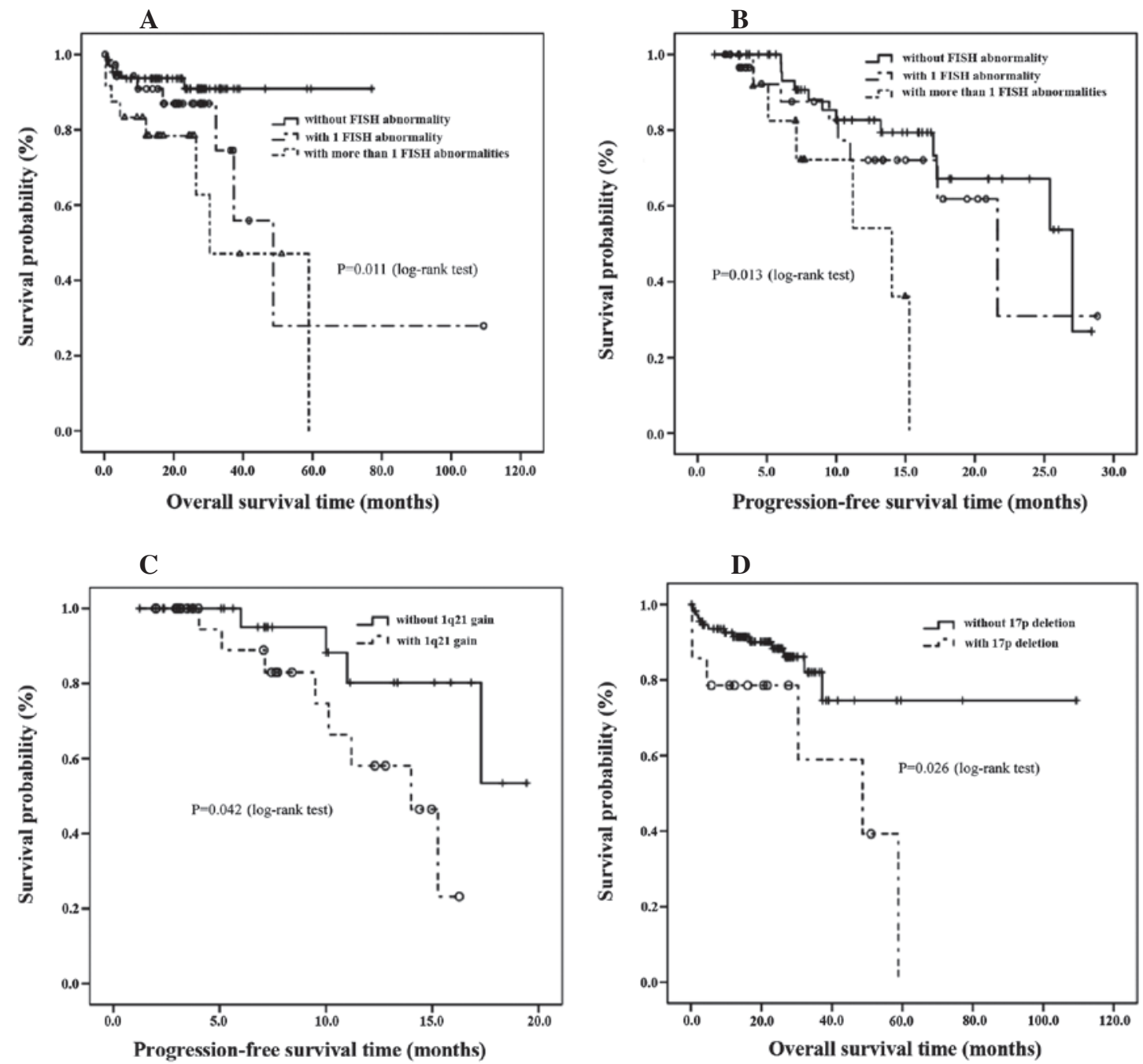

Figure 1. (A) Overall survival (OS) times of multiple myeloma (MM) patients according to fluorescence in situ hybridization (FISH) abnormalities. (B) Progression-free survival (PFS) times of MM patients according to FISH abnormalities. (C) PFS times of MM patients according to 1q21 gain. (D) OS times of MM patients according to $17 \mathrm{p}$ deletion.

gain (82.4 vs. $77.5 \%$; $P=0.605)$, respectively. These results indicate that the treatment response of the patients with $\mathrm{MM}$ was not affected by the presence/absence of the iFISH abnormalities under investigation.

However, in the iFISH-negative group, the patients who received bortezomib-based combination chemotherapy exhibited a significantly higher ORR (95.3 vs. $68.2 \%$; $\mathrm{P}=0.009)$ and a higher $\geq$ VGPR (60.1 vs. $13.6 \%$; $\mathrm{P}=0.001)$ compared with the patients who received the non-bortezomib regimens. Furthermore, for the patients with $\mathrm{t}(4 ; 14)$, the bortezomib-based combination improved the rate of $\geq$ VGPR (100.0 vs. $0.0 \%$; $\mathrm{P}=0.001$; Table II).

Correlation between iFISH abnormalities and patient outcome. The prognostic impact of each iFISH abnormality on the PFS and OS times was analyzed. Patients with two or more iFISH abnormalities had significantly shorter OS and PFS times than patients with one or no abnormality (median OS time, 30.4 vs. 48.7 months vs. not reached (OS time>follow-up time), respectively; $\mathrm{P}=0.013$; Fig. $1 \mathrm{~A}$; and median PFS time, 14.0 vs. 21.6 vs. 27.0 months, respectively; $\mathrm{P}=0.011$; Fig. $1 \mathrm{~B}$ ). Significantly shorter PFS times were observed in the patients with 1q21 gain versus those without 1q21 gain (median PFS time, 14.0 months vs. not reached, respectively; $\mathrm{P}=0.042$; Fig. 1C). The patients with the $17 \mathrm{p} 13$ deletion had a significantly poorer outcome compared with the patients without the $17 \mathrm{p} 13$ deletion, with a median OS time of 48.7 months versus not reached, respectively $(\mathrm{P}=0.026$; Fig. 1D). The presence/absence of $\mathrm{t}(11 ; 14)$ and $\mathrm{t}(4 ; 14)$ were not associated with a statistically significant effect on the PFS or OS times. All data are summarized in Table III.

Of the patients administered non-bortezomib-based chemotherapy, those with two or more iFISH abnormalities had shorter PFS and OS times than those with one or no abnormality (median PFS time, 7.1 vs. 21.6 vs. 27.0 months, respectively; $\mathrm{P}=0.034$; and median OS time, 30.4 vs. 48.7 months vs. not reached, respectively; $\mathrm{P}=0.245$ ). For the patients who received bortezomib-based regimens, the differences between PFS times for those with two or more iFISH abnormalities and those with one abnormality or no abnormality were not statistically significant (median PFS times, 15.3 months vs. not reached vs. not reached, respectively, $\mathrm{P}=0.237$; median OS times for the three groups were $37.3,58.9$ months and not reached, respectively; $\mathrm{P}=0.225$ ). 
Table III. PFS and OS of multiple myeloma patients according to cytogenetic abnormalities.

\begin{tabular}{|c|c|c|c|c|c|c|c|}
\hline & & & PFS time & & & OS time & \\
\hline & $\mathrm{n}$ & $\begin{array}{l}\text { Median, } \\
\text { months }\end{array}$ & $\begin{array}{l}\text { Three-year } \\
\text { estimate, \% } \\
(\text { mean } \pm \mathrm{SD})\end{array}$ & P-value & $\begin{array}{l}\text { Median, } \\
\text { months }\end{array}$ & $\begin{array}{l}\text { Three-year } \\
\text { estimate, } \% \\
(\text { mean } \pm \text { SD) }\end{array}$ & P-value \\
\hline Overall & & & & 0.011 & & & 0.013 \\
\hline 2 or more abnormalities & 24 & 14.0 & $0.0 \pm 0.0$ & & 30.4 & $47.1 \pm 17.9$ & \\
\hline 1 abnormality & 37 & 21.6 & $30.9 \pm 22.8$ & & 48.7 & $74.5 \pm 12.7$ & \\
\hline Normal FISH & 66 & 27.0 & $62.7 \pm 9.7$ & & NR & $90.9 \pm 4.0$ & \\
\hline $\mathrm{t}(11 ; 14)$ & & & & 0.839 & & & 0.289 \\
\hline Positive & 14 & 15.3 & $0.0 \pm 0.0$ & & 30.4 & $38.1 \pm 27.6$ & \\
\hline Negative & 66 & 17.3 & $39.9 \pm 17.8$ & & NR & $75.1 \pm 9.6$ & \\
\hline $\mathrm{t}(4 ; 14)$ & & & & 0.975 & & & 0.588 \\
\hline Positive & 9 & 17.3 & $46.7 \pm 22.6$ & & 58.9 & $59.3 \pm 25.2$ & \\
\hline Negative & 118 & 25.4 & $76.5 \pm 5.4$ & & NR & $90.0 \pm 2.9$ & \\
\hline $17 \mathrm{p}$ deletion & & & & 0.760 & & & 0.026 \\
\hline Positive & 14 & 21.6 & $0.0 \pm 0.0$ & & 48.7 & $58.9 \pm 18.9$ & \\
\hline Negative & 113 & 25.4 & $31.7 \pm 14.9$ & & NR & $91.4 \pm 2.7$ & \\
\hline 1q21 gain & & & & 0.042 & & & 0.885 \\
\hline Positive & 34 & 14.0 & $23.2 \pm 18.1$ & & 30.4 & $44.9 \pm 21.3$ & \\
\hline Negative & 46 & NR & $53.5 \pm 22.9$ & & NR & $79.8 \pm 8.0$ & \\
\hline
\end{tabular}

PFS, progression-free survival; OS, overall survival; FISH, fluorescence in situ hybridization; NR, not reached; SD, standard deviation.

Thus, bortezomib appears to partially overcome the adverse effect caused by iFISH abnormalities.

\section{Discussion}

The present retrospective study included a series of 131 patients with MM who were analyzed for genomic aberrations and were treated with bortezomib/non-bortezomib-based therapies. The incidence of translocation $\mathrm{t}(4 ; 14)$ in the present study $(6.9 \%)$ was marginally lower than that reported by Fonseca et al (12.7\%) (9) and that observed by Avet-Loiseau et al (14\%) (6). The incidence of $\mathrm{t}(14 ; 16)$ in the present study was rare, detected in only one case out of 131 myeloma patients, which may be due to the small sample size. The observed frequencies of $1 \mathrm{q} 21$ gain, $\mathrm{t}(11 ; 14)$ and $17 \mathrm{p} 13$ deletion in the present study were consistent with results published in previous studies $(6,9,23)$. Of the 61 patients with abnormal iFISH results in the present study, $24(39.3 \%)$ had two or more iFISH abnormalities. These results reflect the fact that myeloma cells are characterized by complex genetic abnormalities.

The most extensively employed prognostic system in myeloma is the ISS, a system which stratifies patients into three groups, as determined by serum albumin and $\beta 2$-microglobulin levels. These are factors that are reflective of patient and tumor characteristics, with $\beta 2$-microglobulin an indicator of tumor bulk and renal function, and albumin associated with the patient's general state. In the present study, the ISS stage was not affected by the presence or absence of any known iFISH abnormality, although patients with $\mathrm{t}(4 ; 14)$ had significantly lower albumin levels than patients who lacked $t(4 ; 14)$.

ORR analysis did not reveal statistical differences between the patients with and without iFISH abnormalities. However, in the patients with normal iFISH results, bortezomib-based therapies significantly improved the ORR (95.3 vs. $68.2 \%$ ) and $\geq$ VGPR (60.1 vs. $13.6 \%$ ) compared with the patients receiving non-bortezomib-based therapies. However, for the patients with iFISH abnormalities, bortezomib-based therapies were not shown to be more effective, with the exception that these therapies improved the $\geq$ VGPR (100.0 vs. $0.0 \%)$ for the patients with $\mathrm{t}(4 ; 14)$.

In the present study, the presence of two or more iFISH abnormalities remained the most important unfavorable prognostic factor for patients with MM. The median PFS time of the patients with two or more iFISH abnormalities was found to be only 14.0 months and the OS time reached only 30.4 months; a finding consistent with that of a previous study, which demonstrated that patients with complex karyotypes had the worst outcome (23). The gain of 1q21 has been reported to be an adverse prognostic factor in newly diagnosed or relapsed MM in several studies $(4,5,24)$. In the present study, 1q21 gain, examined by interphase iFISH in 80 patients with myeloma, exerted a marked effect on prognosis. Gain of the 1q21 locus reduced the PFS time to 14.0 months, the same time period as that found in the patients with two or more iFISH abnormalities. However, 1q21 gain was not confirmed as a prognostic factor for OS time $(\mathrm{P}=0.885)$. Deletion and mutation of the p53 tumor suppressor gene, located in 17 p13, are commonly 
observed in patients with MM. The 17p13 deletion is detected in $\sim 10 \%$ patients with MM at the time of diagnosis and this abnormality frequently occurs together with other aberrations (25). Mutation and deletion of the p53 tumor suppressor gene have been markedly associated with a lower likelihood of a response to therapy, and have been revealed as independent prognostic markers for shorter survival times in MM patients $(16,25-27)$. In the present study, which sampled $131 \mathrm{MM}$ cases, patients with the $17 \mathrm{p} 13$ deletion exhibited a significantly shorter OS time than patients without this abnormality (48.7 months vs. not reached; $\mathrm{P}=0.026$ ). Furthermore, out of the 14 patients with the $17 \mathrm{p} 13$ deletion, six (42.9\%) also had a 1q21 gain. However, the prognosis in this group was not significantly different compared with the prognosis of patients who only had the $17 \mathrm{p} 13$ deletion. Other genomic changes, such as $t(4 ; 14)$ and $t(11 ; 14)$, had less effect on PFS and OS times. Previous studies have reported that $t(4 ; 14)$ is an unfavorable prognostic factor $(20,28)$. The results of the present study suggest that patients with $t(4 ; 14)$ had a shorter PFS and OS times than patients who lacked $t(4 ; 14)$ (PFS, 17.3 vs. 25.4 months; OS, 58.9 months vs. not reached); however, this difference was not statistically significant. The lack of a statistically significant difference may be due to the heterogeneity of $\mathrm{t}(4 ; 14)$, or the limited sample size and low number of follow-ups in the present study. Similarly, $t(11 ; 14)$ did not affect the survival outcome, as suggested by several other studies $(6,29,30)$.

Previous clinical trials have demonstrated that bortezomib-based regimens are effective in the treatment of MM and are able to overcome the unfavorable effect of several abnormalities [del (13q), $\mathrm{t}(4 ; 14), \mathrm{t}(14 ; 16)$, del (p53) and 1q21 gain] (31-35). In the present study, the patients with two or more iFISH abnormalities had significantly shorter PFS times than the patients with less or no iFISH abnormality following administration of non-bortezomib-based regimens. However, when the patients received bortezomib-based regimens, the PFS times of the patients with two or more iFISH abnormalities, one abnormality and no abnormality were not significantly different. Thus, bortezomib may improve the adverse outcomes caused by iFISH abnormalities.

In conclusion, the present study demonstrated that patients with two or more iFISH abnormalities, gain of the 1q21 region or deletion of $17 \mathrm{p} 13$ were more likely to exhibit a poor prognosis for $\mathrm{MM}$, and that bortezomib improves the outcome for MM patients with unfavorable iFISH abnormalities. Due to the limited sample size and follow-ups, larger prospective trials are required for a more reliable result.

\section{Acknowledgements}

This study was supported by the National Natural Science Foundation of China (General Program; grant no. 81172252).

\section{References}

1. Ludwig H, Durie BG, Bolejack V, et al: Myeloma in patients younger than age 50 years presents with more favorable features and shows better survival: an analysis of 10,549 patients from the International Myeloma Working Group. Blood 111: 4039-4047, 2008.

2. Raab MS, Podar K, Breitkreutz I, Richardson PG and Anderson KC: Multiple myeloma. Lancet 374: 324-339, 2009.
3. Yeung $\mathbf{J}$ and Chang $\mathrm{H}$ : Genomic aberrations and immunohistochemical markers as prognostic indicators in multiple myeloma. J Clin Pathol 61: 832-836, 2008.

4. Hanamura I,Stewart JP,Huang Y, et al: Frequent gain of chromosome band 1q21 in plasma-cell dyscrasias detected by fluorescence in situ hybridization: incidence increases from MGUS to relapsed myeloma and is related to prognosis and disease progression following tandem stem-cell transplantation. Blood 108: 1724-1732, 2006.

5. Fonseca R, Van Wier SA, Chng WJ, et al: Prognostic value of chromosome 1q21 gain by fluorescent in situ hybridization and increase CKS1B expression in myeloma. Leukemia 20: 2034-2040, 2006.

6. Avet-Loiseau H, Attal M, Moreau P, et al: Genetic abnormalities and survival in multiple myeloma: the experience of the Intergroupe Francophone du Myélome. Blood 109: 3489-3495, 2007.

7. Zojer N, Königsberg R, Ackermann J, et al: Deletion of 13q14 remains an independent adverse prognostic variable in multiple myeloma despite its frequent detection by interphase fluorescence in situ hybridization. Blood 95: 1925-1930, 2000.

8. Keats JJ, Reiman T, Maxwell CA, et al: In multiple myeloma, $\mathrm{t}(4 ; 14)(\mathrm{p} 16 ; \mathrm{q} 32)$ is an adverse prognostic factor irrespective of FGFR3 expression. Blood 101: 1520-1529, 2003.

9. Fonseca R, Blood E, Rue M, et al: Clinical and biologic implications of recurrent genomic aberrations in myeloma. Blood 101: 4569-4575, 2003.

10. Chang H, Sloan S, Li D, et al: The t $(4 ; 14)$ is associated with poor prognosis in myeloma patients undergoing autologous stem cell transplant. Br J Haematol 125: 64-68, 2004.

11. Gertz MA, Lacy MQ, Dispenzieri A, et al: Clinical implications of $\mathrm{t}(11 ; 14)(\mathrm{q} 13 ; \mathrm{q} 32), \mathrm{t}(4 ; 14)(\mathrm{p} 16.3 ; \mathrm{q} 32)$, and $-17 \mathrm{p} 13$ in myeloma patients treated with high-dose therapy. Blood 106: 2837-2840, 2005.

12. Ross FM, Ibrahim AH, Vilain-Holmes A, et al; UK Myeloma Forum: Age has a profound effect on the incidence and significance of chromosome abnormalities in myeloma. Leukemia 19: 1634-1642, 2005.

13. Avet-Loiseau H, Malard F, Campion L, et al; Intergroupe Francophone du Myélome: Translocation $\mathrm{t}(14 ; 16)$ and multiple myeloma: is it really an independent prognostic factor? Blood 117: 2009-2011, 2011

14. Drach J, Ackermann J, Fritz E, et al: Presence of a p53 gene deletion in patients with multiple myeloma predicts for short survival after conventional-dose chemotherapy. Blood 92: 802-809, 1998.

15. Chang H, Qi C, Yi QL, Reece D and Stewart AK: p53 gene deletion detected by fluorescence in situ hybridization is an adverse prognostic factor for patients with multiple myeloma following autologous stem cell transplantation. Blood 105: 358-360, 2005.

16. Avet-Loiseau H, Leleu X, Roussel M, et al: Bortezomib plus dexamethasone induction improves outcome of patients with $\mathrm{t}(4 ; 14)$ myeloma but not outcome of patients with del(17p). J Clin Oncol 28: 4630-4634, 2010.

17. Chang H, Trieu Y, Qi X, Xu W, Stewart KA and Reece D: Bortezomib therapy response is independent of cytogenetic abnormalities in relapsed/refractory multiple myeloma. Leuk Res 31: 779-782, 2007.

18. Pineda-Roman M,Zangari M,Haessler J,et al: Sustained complete remissions in multiple myeloma linked to bortezomib in total therapy 3: comparison with total therapy 2. Br J Haematol 140: 625-634, 2008

19. Grogan TM, Van Camp B and Kyle RA: Plasma cell neoplasms. In: World Health Organization Classification of Tumors. Pathology and Genetics of Tumours of Haematopoietic and Lymphoid Tissues. Jaffe ES, Harris NL, Stein H and Vardiman JW (eds). IARC Press, Lyon, pp144, 2001.

20. Greipp PR, San Miguel J, Durie BG, et al: International staging system for multiple myeloma. J Clin Oncol 23: 3412-3420, 2005.

21. Durie BG and Salmon SE: A clinical staging system for multiple myeloma. Correlation of measured myeloma cell mass with presenting clinical features, response to treatment, and survival. Cancer 36: 842-854, 1975.

22. Durie BG, Harousseau JL, Miguel JS, et al; International Myeloma Working Group: International uniform response criteria for multiple myeloma. Leukemia 20: 1467-1473, 2006.

23. Nemec P, Zemanova Z, Kuglik P, et al; Czech Myeloma Group: Complex karyotype and translocation $\mathrm{t}(4 ; 14)$ define patients with high-risk newly diagnosed multiple myeloma: results of CMG2002 trial. Leuk Lymphoma 53: 920-927, 2012.

24. Grzasko N, Hus M, Pluta A, et al: Additional genetic abnormalities significantly worsen poor prognosis associated with 1q21 amplification in multiple myeloma patients. Hematol Oncol 31: 41-48, 2013. 
25. Neben K, Jauch A, Bertsch U, et al: Combining information regarding chromosomal aberrations $\mathrm{t}(4 ; 14)$ and $\operatorname{del}(17 \mathrm{p} 13)$ with the International Staging System classification allows stratification of myeloma patients undergoing autologous stem cell transplantation. Haematologica 95: 1151-1157, 2010.

26. Chang $\mathrm{H}$, Yeung J, Qi $\mathrm{C}$ and $\mathrm{Xu} \mathrm{W}$ : Aberrant nuclear p53 protein expression detected by immunohistochemistry is associated with hemizygous p53 deletion and poor survival for multiple myeloma. Br J Haematol 138: 324-329, 2007.

27. Xiong W, Wu X, Starnes S, et al: An analysis of the clinical and biologic significance of TP53 loss and the identification of potential novel transcriptional targets of TP53 in multiple myeloma. Blood 112: 4235-4246, 2008.

28. Fonseca R, Debes-Marun CS, Picken EB, et al: The recurrent IgH translocations are highly associated with nonhyperdiploid variant multiple myeloma. Blood 102: 2562-2567, 2003.

29. Fonseca R, Blood EA, Oken MM, et al: Myeloma and the $\mathrm{t}(11 ; 14)(\mathrm{q} 13 ; \mathrm{q} 32)$ : evidence for a biologically defined unique subset of patients. Blood 99: 3735-3741, 2002.

30. Stewart AK and Fonseca R: Prognostic and therapeutic significance of myeloma genetics and gene expression profiling. J Clin Oncol 23: 6339-6344, 2005.
31. San Miguel JF, Schlag R, Khuageva NK, et al; VISTA Trial Investigators: Bortezomib plus melphalan and prednisone for initial treatment of multiple myeloma. N Engl J Med 359: 906-917, 2008

32. Barlogie B, Anaissie E, van Rhee F, et al: Incorporating bortezomib into upfront treatment for multiple myeloma: early results of total therapy 3. Br J Haematol 138: 176-185, 2007.

33. Shaughnessy JD, Zhou Y, Haessler J, et al: TP53 deletion is not an adverse feature in multiple myeloma treated with total therapy 3. Br J Haematol 147: 347-351, 2009.

34. Richardson PG, Xie W, Mitsiades C, et al: Single-agent bortezomib in previously untreated multiple myeloma: efficacy, characterization of peripheral neuropathy, and molecular correlations with response and neuropathy. J Clin Oncol 27: 3518-3525, 2009.

35. Chang H, Qi X, Trieu Y, et al: Multiple myeloma patients with CKS1B gene amplification have a shorter progressionfree survival post-autologous stem cell transplantation. Br J Haematol 135: 486-491, 2006. 